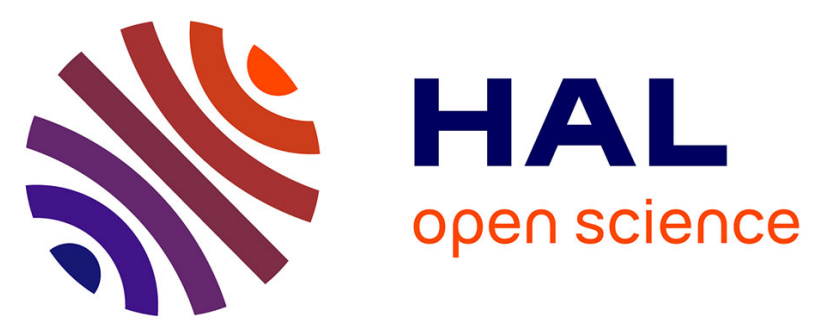

\title{
La viticulture antique dans le Tricastin (moyenne vallée du Rhône)
}

Cécile Jung, Thierry Odiot, Jean-François Berger, Damien Seris, Laurent Bouby, José Antonio López Sáez, Solange Farbos-Texier, Laurence Rousseau

\section{- To cite this version:}

Cécile Jung, Thierry Odiot, Jean-François Berger, Damien Seris, Laurent Bouby, et al.. La viticulture antique dans le Tricastin (moyenne vallée du Rhône). Gallia - Archéologie de la France antique, 2001, La viticulture en Gaule, 58, pp.113-128. 10.3406/galia.2001.3176 . halshs-00204654

\section{HAL Id: halshs-00204654 \\ https://shs.hal.science/halshs-00204654}

Submitted on 20 Jan 2020

HAL is a multi-disciplinary open access archive for the deposit and dissemination of scientific research documents, whether they are published or not. The documents may come from teaching and research institutions in France or abroad, or from public or private research centers.
L'archive ouverte pluridisciplinaire HAL, est destinée au dépôt et à la diffusion de documents scientifiques de niveau recherche, publiés ou non, émanant des établissements d'enseignement et de recherche français ou étrangers, des laboratoires publics ou privés.

\section{(ㅇ)(1) $\$$}

Distributed under a Creative Commons Attribution - NonCommercial - NoDerivatives| 4.0 


\title{
LA VITICULTURE ANTIQUE DANS LE TRICASTIN (MOYENNE VALLÉE DU RHÔNE)
}

\author{
Cécile Jung, Thierry Odiot, Jean-François Berger, Damien Seris \\ avec la collaboration de Laurent BouBY, Antonio LoPEZ SAEZ, \\ Solange FARBOS-TEXIER et Laurence RouSSEAU
}

\begin{abstract}
Mots-clés. Vallée du Rhône, Drôme, Tricastin, cadastre B d'Orange, Augusta Tricastinorum, Saint-Paul-Trois-Châteaux, Donzère, villae du Molard, du Fayn, de Saint-Vincent et de Termini, fouloirs, pressoirs, cellier, dolia, inscriptions sur dolia, terroirs, fosses de plantation.

Key-words. Rhône valley, Drôme, Tricastin, Orange B centuriation, Augusta Tricastinorum, Saint-Paul-Trois-Châteaux, Donzère, Le Molard, Le Fayn, Saint-Vincent and Termini Roman villae, wine-presses, wine-cellars, dolia, inscriptions on dolia, vineyards, planting holes.

Résumé. En moyenne vallée du Rhône, le Tricastin à livré de nombreux indices de viticulture antique. Plusieurs villae viticoles possèdent des fouloirs, des pressoirs et des celliers. La plus importante, celle du Molard, est dotée de deux fouloirs, qualre pressoirs et d'un cellier d'une contenance de 2500 hectolitres. Outre ces exploitations reconnues par les fouilles, les prospections archéologiques et surtout l'archéologie de sauvetage ont livré des traces plus fugaces comme des fosses de plantations. Tous ces indices sont corroborés par les données de la paléobotanique. Ces observations permettent de débattre sur le problème de la superficie el du rendement des domaines, ainsi que sur celui des circuits commerciaux qui expliquent la présence dans ce secteur d'une production viticole de masse pendant la seconde moitié du $I^{e r}$ s. et au début du II s. après J.-C.
\end{abstract}

\begin{abstract}
In the central part of the Rhône valley, the Tricastin offers several occurrences of Roman viticulture. Several Roman villae had wine-presses and important wine-cellars. Among these the Molard villa, the largest one, had wine-presses and a cellar which contained $2500 \mathrm{hl}$ of wine. In this area, excavations, surveys and rescue archaeology reveal other evidences such as planting holes. These occurrences allow us to discuss the question of the size of the estates and the commercial links. The Tricastin is known for its mass production of wine during the second half of the $1^{\text {rst }}$ century $A D$ and the beginning of the $2^{\text {nd }}$ century.
\end{abstract}

La façade rhodanienne du Tricastin correspond à une très basse plaine alluviale. Située en rive gauche du Rhône, elle s'étend entre le cours du Jabron qui traverse le bassin de Montélimar au nord et le Lez qui la limite au sud de la plaine de Pierrelatte. Son évolution géomorphologique à l'Holocène explique la grande variété de sols reconnus et cartographiés aujourd'hui (Bornand, 1972 ; Tounsi et al., 1997).

Cette plaine est limitée par le cours du Rhône à l'ouest, et à l'est par une série de reliefs tertiaires et secondaires qui forme une limite topographique (massif
d'Uchaux, collines de Saint-Paul-Trois-Châteaux, de La Garde-Adhémar et plateau de Montjoyer-Aleyrac) (fig. 51).

Au sein de cet espace géographique, les données sur la viticulture antique demeurent hétérogènes. En effet, si les travaux réalisés depuis quelques années sur la plaine de Pierrelatte nous permettent d'avoir désormais une connaissance fine du terroir et de son évolution, en revanche, les données concernant la mise en culture dans le sud du bassin de Montélimar demeurent encore sporadiques. 


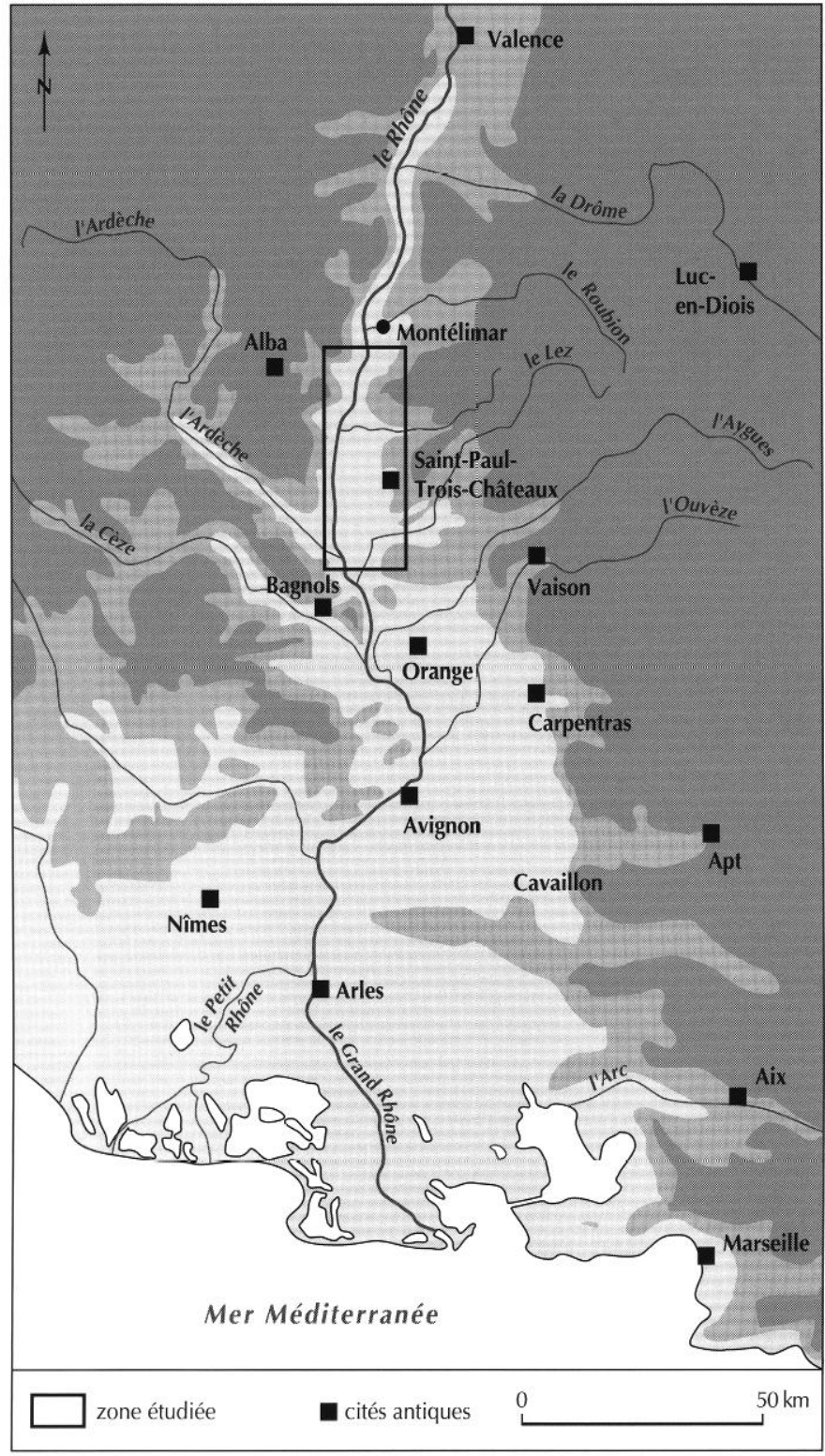

Fig. 51 - Cadre géographique de la zone étudiée.

Afin de mieux comprendre l'essor puis le déclin de la viticulture antique dans le Tricastin, il faut d'abord présenter les grandes phases de la dynamique du peuplement de ce secteur ${ }^{141}$. Elles s'apparentent à celles observées dans les autres régions de la basse vallée du Rhône, avec un léger décalage dans le temps au niveau

141. Celles-ci ont pu être analysées grâce aux travaux réalisés dans le cadre du programme curopécn "Archacomedes "financé par la commission DGXII (climat et risques naturels) de l'Union européenne.

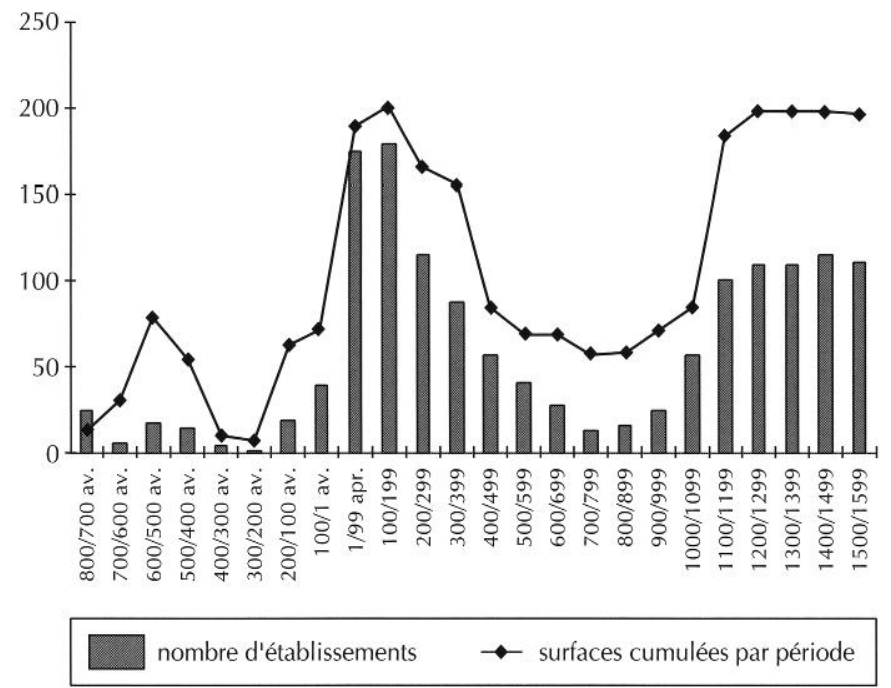

Fig. 52 - Hislogramme représentant la dynamique du peuplement en Tricastin associée à la courbe des superficies occupées (cf. Archaeomedes, II).

des créations de sites ou des abandons (Archaeomedes, 1998).

En effet, le nombre de créations de sites augmente dans la seconde moitié du $\mathrm{I}^{\mathrm{er}} \mathrm{s}$. après J.-C., ce qui paraît relativement tardif par rapport aux régions plus méridionales qui connaissent un essor dans la création de sites dès le début du $\mathrm{I}^{e r} \mathrm{~s}$ (fig. 52). C'est sous les Flaviens que l'occupation du territoire est la plus dense ${ }^{142}$ et les structures administratives, économiques et juridiques finissent de se mettre en place. La révision du plan cadastral d'Orange sous Vespasien en 77 après J.-C. en est une illustration.

De nombreuses traces d'occupation correspondent souvent à de petits établissements interprétés comme des annexes agraires (Archaeomedes, 1998). Dans le courant du $\mathrm{II}^{\mathrm{c}} \mathrm{s}$., on note de nombreux abandons de sites ainsi qu'un déficit de créations de sites qui marquent un changement majeur dans l'occupation du territoire (fig. 52). On observe en effet l'abandon des sites de petite taille alors que la plupart des « grands » sites, présentant des éléments de confort (mosaïques, tubuli, enduits peints) et implantés à proximité d'axes de circulation, perdurent au cours de l'Antiquité tardive (IV $-\mathrm{VI}^{\mathrm{e}} \mathrm{s}$.).

142. La densification de l'habitat dispersé complété par un maillage dense d'agglomérations secondaires s'intègre dans la centuriation parachevant la mise en place d'une nouvelle structuration économique du territoire de la cité. 
Dans le Tricastin, ainsi que dans d'autres régions de la vallée du Rhône (comme les Alpilles, le Haut-Comtat ou le Beaucairois), les fluctuations du nombre de sites sont mises en rapport avec une économie basée sur certaines cultures spéculatives (viticulture, oléiculture). Les établissements trop spécialisés sont donc rapidement abandonnés, dès lors que le marché est moins soutenu (Archaeomedes, 1998, p. 83).

Une série de fouilles de sauvetage et des prospections systématiques complétées par des sondages ont permis d'identifier des centres de production viticole qui s'inscrivent dans cette dynamique économique.

\section{LES CENTRES DE PRODUCTION ET DE TRANSFORMATION}

\section{LA VILLA DU MOLARD À DONZÈRE (DRÔME)}

Le site du Molard est implanté à $15 \mathrm{~km}$ au nord-ouest d'Augusta Tricastinorum (Saint-Paul-Trois-Châteaux), capitale des Tricastins (fig. 53). Il est situé à proximité de l'agglomération antique de Donzère probablement dotée d'un port sur le Rhône ${ }^{143}$. Les premières traces d'occupation sont datées de la période augustéenne sans qu'une fonction puisse être attribuée aux structures ${ }^{144}$.

C'est au milieu du I ${ }^{\mathrm{er}} \mathrm{s}$. qu'est implanté un ensemble de bâtiments couvrant $10000 \mathrm{~m}^{2}$. Ces bâtiments sont construits en une seule phase, selon un programme architectural homogène sous-tendu par une conception fonctionnelle de l'utilisation des espaces de travail et de circulation. La totale symétrie du plan et l'utilisation de modules garantissaient aux utilisateurs des axes de circulation et une bonne organisation des espaces de travail.

Les bâtiments sont divisés en deux parties (fig. 54) :

- au nord, deux blocs (T, E, O) encadrent symétriquement un grand entrepôt (pièces $F$ ) bordé d'une galerie (G) ; il s'agit de la pars fructuaria ${ }^{145}$.

143. Celui-ci est mentionné en 840 . Il est situé au débouché du défilé de Donzère, point de rupture de charge sur le Rhône.

144. Une description détaillée de cet établissement a été publiée par T. Odiot (1996).

145. La spécialisation de cette partie du bâtiment, uniquement consacrée à la vinification, pourrait permettre d'employer le terme de pars fructuaria selon la définition de Columelle, I, 6, v. 1-21: " Modus autem memborumque nimerus aptetur universo consaepto et dividatur in tres partes, urbanam, rusticam, fructuariam. [...] Pars autem fructuaria dividitur

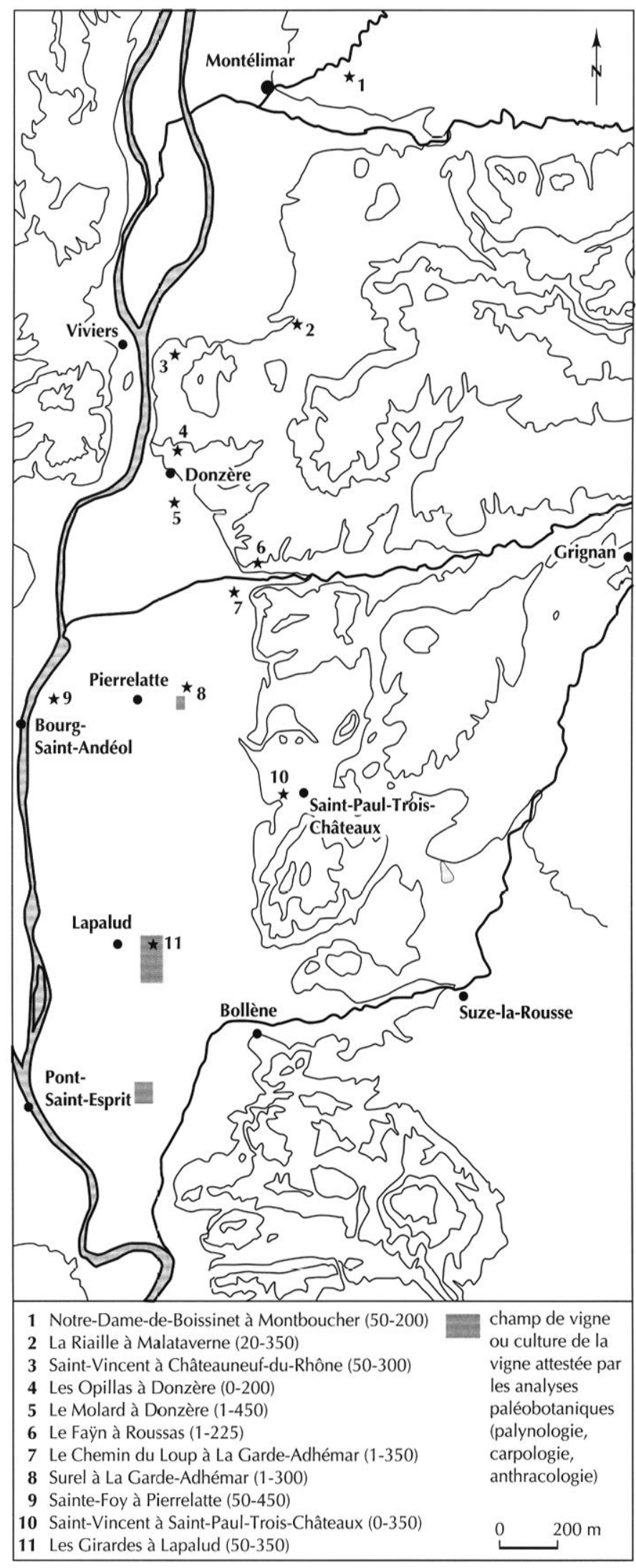

Fig. 53 - Localisation des sites viticoles et des plantations de vignes. 


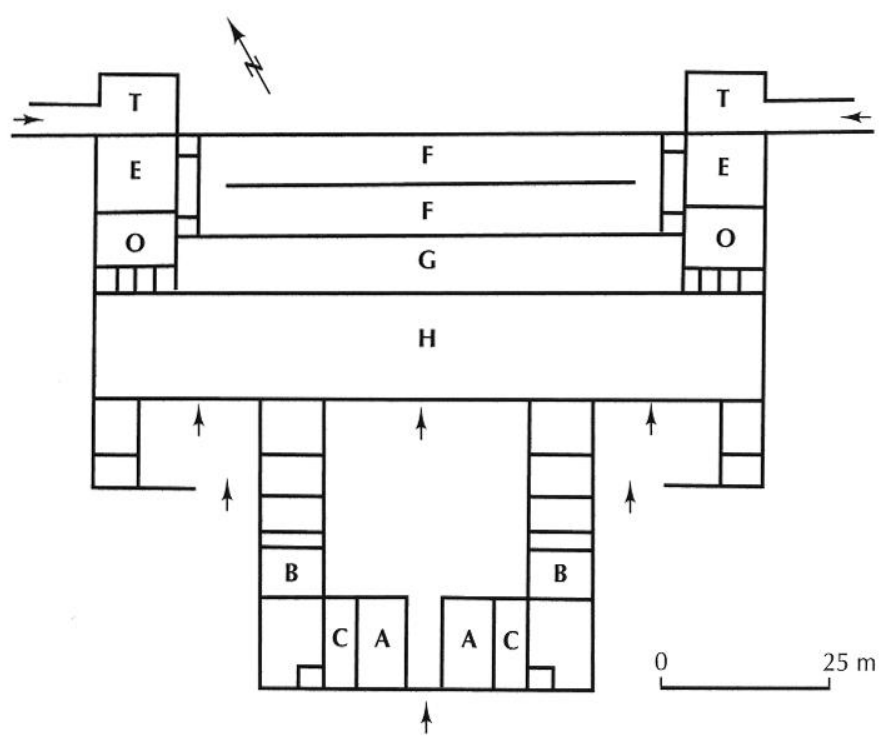

Fig. 54 - Plan de la villa du Molard à Donzère (Drôme).

- au sud, séparé de la partie nord de la villa par une grande cour rectangulaire $(\mathrm{H})$, un ensemble de pièces (B, C, A), moins bien conservées, s'organise, lui aussi, de façon symétrique autour d'une cour et pourrait être interprété comme la pars urbana ou peut-être la pars rustica. La totalité des bâtiments situés dans la partie nord du site est interprétable: aires de pressurage, cellier, galeries de circulation, cour, fontaines et systèmes de gardiennage. En revanche, les logements de la familia, les écuries, étables, râteliers, greniers pour les récoltes des cultures fourragères et vivrières, etc., restent à localiser. La partie sud du site pourrait, par son organisation, remplir cette fonction.

Les chemins d'accès à la villa confirment cette organisation bipolaire. Au sud, deux petits pavillons permettent de contrôler le double accès à la partie d'habitation ${ }^{146}$, tandis qu'au nord, le double accès au cellier est fermé par de lourds ventaux. Cette surveillance des circulations dans la villa et l'isolement du cellier par rapport

partes, urbanam, rusticam, fructuariam. [...] Pars autem fructuaria dividitur in cellam oleariam, torculariam, cellam vinariam, defrutariam, faenolia paleariaque et apothecas et horrea, ut ex iis, quae sunt in plano, custodiam recipiat umidarum rerum tamquam vini aut olei venalium; siccae autem congerantur tabulatis, frumenta, faenum, frondes, palea ceteraque pabula. "

146. Varron, $R . R, I, 13,2$ : "Le fermier (vilicus) doit avoir son logement le plus près possible de la porte d'entrée, et savoir qui entre et qui sort la nuit et ce qu'il a sur lui, surtout s'il n'y a pas de portier. [...] dans l'enceinte des bâtiments couverts assez grands pour les voitures, et le reste du matériel, [...]. » au reste des bâtiments renforcent l'impression qu'il s'agit d'une organisation très hiérarchisée ( $c f$. infra).

\section{LE FOULOIR ET LES PRESSOIRS}

De part et d'autre du cellier, sur ses côtés est et ouest, se trouvent deux structures symétriques très arasées. Un espace central (fouloir), long de $8,80 \mathrm{~m}$ et large de 2,10 m, est flanqué au nord et au sud de cuves latérales carrées (lacus), de 2,10 m de côté. Le sol est constitué par une couche de mortier de tuileau reposant sur un lit de galets damés sur un niveau d'argile. Le fouloir oriental, mieux conservé, a gardé sur les parois de la cuve des traces de mortier de tuileau. Les fouloirs dominaient le cellier comme le préconisera plus tard Palladius ${ }^{147}$. De part et d'autre du cellier, les pièces $E$ et $O$ abritaient chacune deux pressoirs de type catonien (Brun, 1986, p. 225-247), dont le sol était constitué de terrazzo. Au sud de ces pièces, deux groupes de quatre cuves recueillaient le jus de presse.

\section{LE CELIIER}

Le cellier correspond à un grand bâtiment rectangulaire de 67,50 m de long sur 14,90 m de côté. Il est séparé en deux travées symétriques par un mur central porteur de dés de pierre du Midi (molasse miocène de SaintRestitut), espacés de $3,50 \mathrm{~m}$ chacun, qui supportaient des colonnes du même matériau et d'un diamètre de $0,90 \mathrm{~m}$. Chaque travée a été profondément excavée $(1,80 \mathrm{~m})$ et une épaisse couche d'argile nivelle le fond des terrassements et sert d'assise aux dolia. Seuls cinq dolia ont été retrouvés in situ dans la partie fouillée. Mais le décapage de la couche d'argile leur servant d'assise a permis de restituer leur agencement dans le cellier. Les jarres sont alignées, à raison de trois rangées par travée, permettant ainsi d'installer 200 dolia au total dans le cellier.

147. " Cellam vinariam septemtrioni habere debemus opposilam, frigidam vel obscurae proximam, longe a balneis, stabulis, furno, sterculinis, cisternis, aquis et ceteris odoris horrendi, ita instruclam necessariis, vt non vincatur a fructv, sic avtem dispositam, ut basilicae ipsivs forma calcatorivm loco habeat altiore constvctum, ad quod, inter dvos lacos qui ad excipienda vina hinc inde depressi sint, gradibus tribus fere ascendatur avt quatvor; ex his lacvbos canales strvcti vel tubi fictiles circa extremos parietes currant et svbiectis lateri svo doliis per vicinos maetvs manantia vian defuvntant. "(Palladius, Traité d'agriculture, I, 18). 
Après la mise en place des dolia, l'ensemble du cellier a été remblayé de façon à ce que les sols de circulation (constitués de terrazzo) atteignent les trois quarts de la hauteur des jarres. Au niveau de chaque travée de dolia, un espacement d'environ $0,30 \mathrm{~m}$ permettait de circuler entre les ouvertures des vases ${ }^{143}$. Dans l'axe central, de part et d'autre de la colonnade, tout comme aux extrémités est et ouest, une banquette large de $2 \mathrm{~m}$ assurait un espace de travail et de circulation. Ce n'est qu'après ces étapes du chantier que l'on a construit les murs et la toiture.

Les murs étaient recouverts par un enduit mural rouge en plinthe (hauteur : $0,80 \mathrm{~m}$ ) et uniformément blanc en élévation. Si le cellier semble ne pas avoir comporté d'ouverture de plain-pied au nord, trois ouvertures (au minimum) donnant sur la galerie sont restituables sur le côté sud du bâtiment. À l'est et à l'ouest, les fouloirs dominent le cellier et ouvrent l'espace vers les pressoirs.

\section{LES DOLIA}

Les mesures effectuées sur les dolia permettent de restituer leur taille et leur capacité comprise entre 1200 et 1500 litres. Une série d'inscriptions en urnae imprimées sur les rebords de ces dolia avant cuisson corrobore ces estimations : $X C V=95$ urnae et $2 / 12$, soit 1 244,7 litres. La capacité maximale du cellier devait donc avoisiner 2500 hectolitres. Par ailleurs, l'analyse chromatographique en phase gazeuse, effectuée par Françoise Formenti (université catholique de Lyon) sur les dépôts noirs reconnus sur la partie interne des dolia, atteste la présence de poix. Ces éléments et l'organisation générale des lieux confirment donc la fonction vinicole de l'établissement.

\section{NATURE DU SITE}

$\mathrm{Au}$ sud du cellier, une galerie ouvre sur une grande cour rectangulaire dotée sur les côtés de deux abreuvoirs. Deux portes charretières donnent accès à l'extérieur du site et une porte centrale permet d'accéder directement de la cour vers les bâtiments de la partie sud

148. Géop., VI, 3, 10-11: «Les jarres ne devront pas se toucher; on laissera entre elles une distance d'un pied pour permettre aux employés du cellier l'accès aux jarres de l'intérieur et pour éviter qu'une jarre qui tourne ne gâte les voisines. ” du site. Celle-ci est constituée de deux bâtiments en « $L$ » symétriquement inversés. L'aile ouest est composée d'une série de six pièces construites d'un seul tenant subdivisant un corps de bâtiment long de $40 \mathrm{~m}$ et large de $10 \mathrm{~m}$. La fonction des pièces reste impossible à déterminer pour ce bâtiment qui comportait un étage. Au sud, une cave s'inscrit dans l'aile orientale du bâtiment.

Bien isolés de la partie viticole du site par une série de portes et un système de gardiennage, les bâtiments sud de la villa restent difficiles à interpréter. Rien ne prouve que la maison du maître se trouvait dans cet ensemble ${ }^{149}$. L'absence d'une pars urbana agrémentée de signes de richesse, comme on aurait pu l'attendre, peut traduire le rôle particulier du site, qui serait seulement doté d'une pars rustica.

Dans ce contexte, la proximité du cours du Rhône permet d'interpréter Le Molard comme un centre de production tourné directement vers l'exportation.

Le site est contemporain de la phase de multiplication des ateliers d'amphores gauloises en Narbonnaise, à partir du milieu du $\mathrm{I}^{\mathrm{er}}$ s. de notre ère (Tchernia, 1986a). Il n'est donc pas surprenant qu'un investisseur cherchant un profit sur ce marché en plein développement s'installe dans la région à proximité d'un point de rupture de charge sur le Rhône.

En ce qui concerne la durée de fonctionnement du site vinicole, le matériel céramique retrouvé dans les niveaux de destruction permet d'affirmer que, dans le dernier tiers du II $^{\mathrm{e}}$ s., l'établissement connaît d'importantes modifications. Au sud, la cave est remblayée avec de nombreux éléments de démolition, montrant que des murs et des toitures sont détruits ; dans le cellier, les dolia sont récupérés. L'ensemble du cellier est ensuite nivelé pour laisser place à un sol qui sera occupé durant l'Antiquité tardive. Cette destruction du cellier est contemporaine de la phase de raréfaction des indices de viticulture dans le Tricastin.

\section{LA VILLA DU FAYN À ROUSSAS (DRÔME)}

En 1994, une série de sondages a mis au jour le plan partiel d'une villa vinicole dont les vestiges s'étendent sur

149. La présence de tubuli d'hypocauste retrouvés en prospection (dans le secteur non fouillé) ne prouve pas, à elle seule, l'existence d'un bâtiment thermal. 


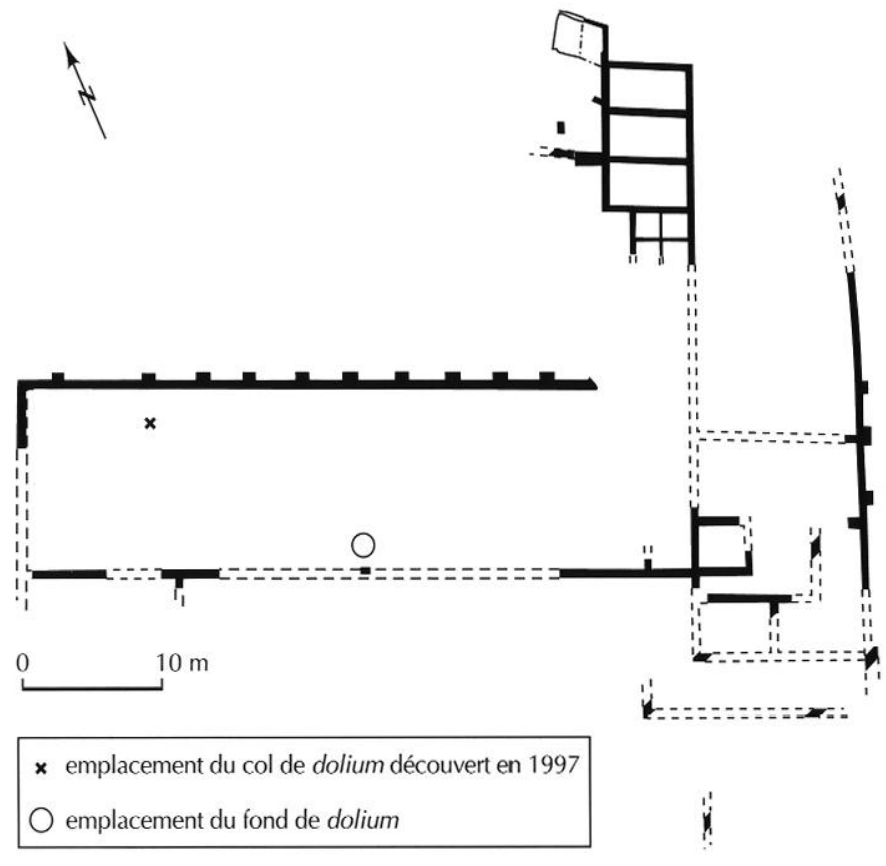

Fig. 55 - Plan de la villa du Fayn à Roussas (Drôme).

$2500 \mathrm{~m}^{2}$ (fig. 55). Implantée sur une terrasse artificielle de $6000 \mathrm{~m}^{2}$, elle s'inscrit au centre de la centurie DDXXIV CKV du cadastre B d'Orange. Au sud, en contrebas des structures archéologiques, subsistent les ruines de la ferme du Fayn, mentionnée sur la carte de Cassini. La villa fut probablement construite à la fin du $\mathrm{I}^{\mathrm{er}} \mathrm{s}$. de notre ère et abandonnée à la fin du $\mathrm{II}^{\mathrm{e}} \mathrm{s}$. ou au début $\mathrm{du} \mathrm{III}^{\mathrm{c}} \mathrm{s}$. Une occupation antérieure, datable du début du $\mathrm{I}^{\text {er }} \mathrm{s}$. de notre ère, a été mise en évidence sans que l'on puisse être plus précis.

Une salle, orientée nord-ouest/sud-cst, de $47,50 \mathrm{~m}$ x 12,30 m, interprétée comme un cellier dont l'extension vers l'est n'a pu être déterminée, abritait des dolia. Le mur nord est rythmé par dix contreforts. Dans le cellier, est conservé en place un fond de dolium, dont l'analyse par $\mathrm{M}^{\mathrm{me}}$ Formenti (ICPI, Lyon) n'a pas révélé la présence d'acide gras. Le quart d'un col de dolium, trouvé en surface à l'emplacement d'une fosse, atteste une vaine tentative de récupération lors de l'abandon du cellier.

En retour d'aile, à l'est du cellier, un ensemble de structures rectangulaires et carrées rappelle des emplacements de cuves, mais aucun élément archéologique n'assure cette identification. Du fait de l'arasement du site, les sondages n'ont pas permis de localiser les fouloirs et les pressoirs.
$\mathrm{Au}$ sud du cellier, une série de murs est édifiée au milieu du II ${ }^{\mathrm{e}}$ s. après J.-C. Le fort pendage nord-est/sudouest implique une construction en terrasse pour venir s'appuyer sur la moyenne terrasse du Riss. Une pièce au sol de terrazzo vient s'accoler au nord-ouest des structures rectangulaires précédentes, mais selon une orientation différente. Ses murs sont décorés d'un enduit peint de couleur rouge. Rien ne permet de rattacher le sol de terrazzo à un secteur d'habitat qui pourrait se situer au sud du cellier, d'autant que des pièces à vocation agricole recouvertes d'enduit peint sont attestées. Une réoccupation sporadique du site eut lieu au IV $\mathrm{s}$.

\section{LE SITE DE TERMINI À MALATAVERNE (DRÔME)}

En 1968, lors de la construction de l'autoroute A7, Claude Latarche est intervenu pour assurer lc dégagement d'une partie d'un édifice gallo-romain (Leglay, 1971). Il faut noter que cette opération fut le seul sauvetage réalisé lors de la construction de cette autoroute dans la région (Latarche, 1969).

Les vestiges, situés à proximité de la voie d'Agrippa à son franchissement de la Riaille, couvraient une superficie de $176 \mathrm{~m}^{2}$ (fig. 53). La végétation et les remblais de l'autoroute rendent difficile une estimation de la surface totale du site, et les observations rapides qui ont pu être faites sur les vestiges posent le problème du statut de cet établissement.

\section{Plan du bâtiment}

Les bâtiments dégagés sont constitués d'une pièce principale de $63 \mathrm{~m}^{2}$ (fig. $56, \mathrm{n}^{\circ} 8$ ), interprétable comme un fouloir avec sa cuve de recueil dont le fond est situé à $1,20 \mathrm{~m}$ en dessous de la margelle. La pièce $\mathrm{n}^{\circ} 6$ correspond à un pressoir avec ses cuves (fig. $56, \mathrm{n}^{\text {os }} 1$ et 2) et il faut probablement localiser la salle de manœuvre à l'ouest des cuves. Il existait une troisième cuve reliée à la cuve n ${ }^{\circ} 2$ par un caniveau de pierre comportant une plaque de plomb. Au sud de ces structures, un chai comprenant un dolium a été partiellement dégagé (fig. 56, $\mathrm{n}^{\circ} 4$ ). La capacité du dolium a été estimée par C. Latarche à environ 1700 litres. Les parois de cette jarre étaient recouvertes de poix, ce qui permit au fouilleur d'envisager une fonction viticole pour le site. À proximité du dolium, le bloc de pierre $\mathrm{n}^{\circ} 5$ pourrait 


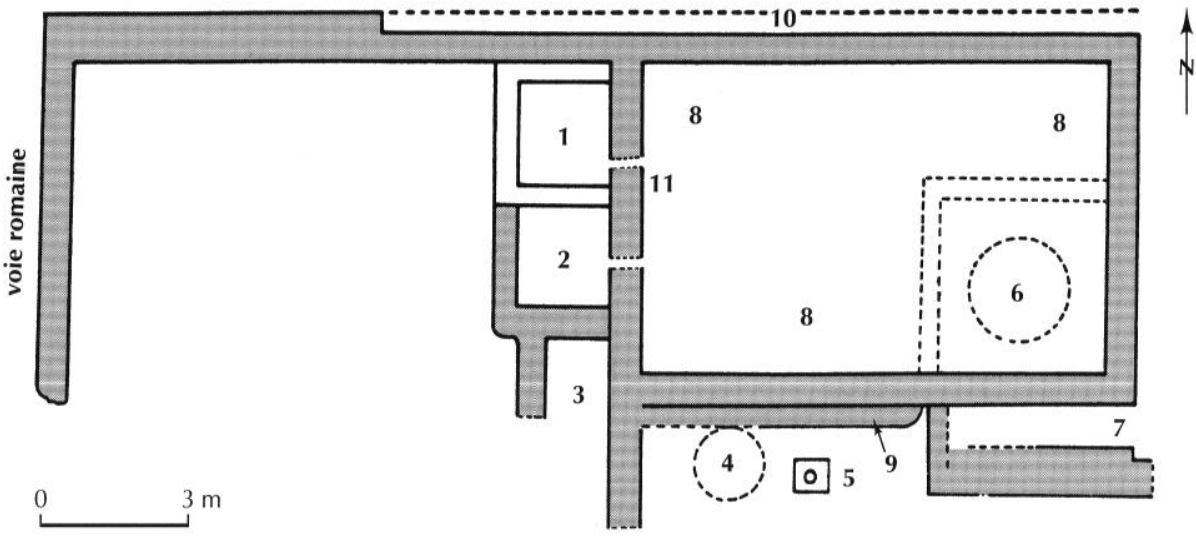

Fig. 56 - Plan de la villa de Termini à

\begin{tabular}{|lrl|}
\hline $\mathbf{1}$ impluvium (transformé en citerne) & $\mathbf{6}$ pièce pavée de mosaïques avec cercle central \\
$\mathbf{2}$ citerne intermédiaire & $\mathbf{7}$ entrée par escalier accédant au perron \\
$\mathbf{3}$ petite citerne & $\mathbf{8}$ & glacis de mortier (recouvrant d'autres mosaïques) \\
$\mathbf{4}$ dolium & $\mathbf{9}$ mur de soutènement de la cave \\
$\mathbf{5}$ pierre du dolium & $\mathbf{1 0}$ contrefort au bas du mur nord \\
& $\mathbf{1 1}$ conduite en tuiles creuses traversant le mur vers la citerne \\
\hline
\end{tabular}
fig. 37).

relation une phase chronologique précise avec un état du bâtiment. On ne peut donc confirmer la datation proposée par le fouilleur qui pensait que l'état viticole de la villa de Malataverne datait du $\mathrm{III}^{\mathrm{e}} \mathrm{s}$. Une datation plus haute est tout à fait envisageable.

\section{LES AUTRES INDICES DE PRÉSENCE DE LA VITICULTURE}

\section{DES DONNÉES DE FOUILLES LACUNAIRES OU EN COURS DE PUBLICATION}

Trois sites fouillés ces dernières années peuvent correspondre à des sites viticoles. L'établissement de SaintVincent à Saint-Paul-Trois-Châteaux (fouille T. Odiot) a livré les vestiges de bassins enduits de mortier hydraulique (Odiot et al. dir., 1992, p. 115-132). Un bâtiment, long de $66,80 \mathrm{~m}$ et large de $16 \mathrm{~m}$, est flanqué à l'ouest de deux bassins hydrauliques. À proximité, une pièce pourrait correspondre à un espace de pressurage. Toutefois l'état d'arasement du site ne permet pas de pousser plus avant l'interprétation des vestiges.

Sur le site des Girardes à Lapalud (Vaucluse) (fouille K. Roger), les probables vestiges d'un pressoir ont été découverts. Cet établissement se trouve à proximité d'un 

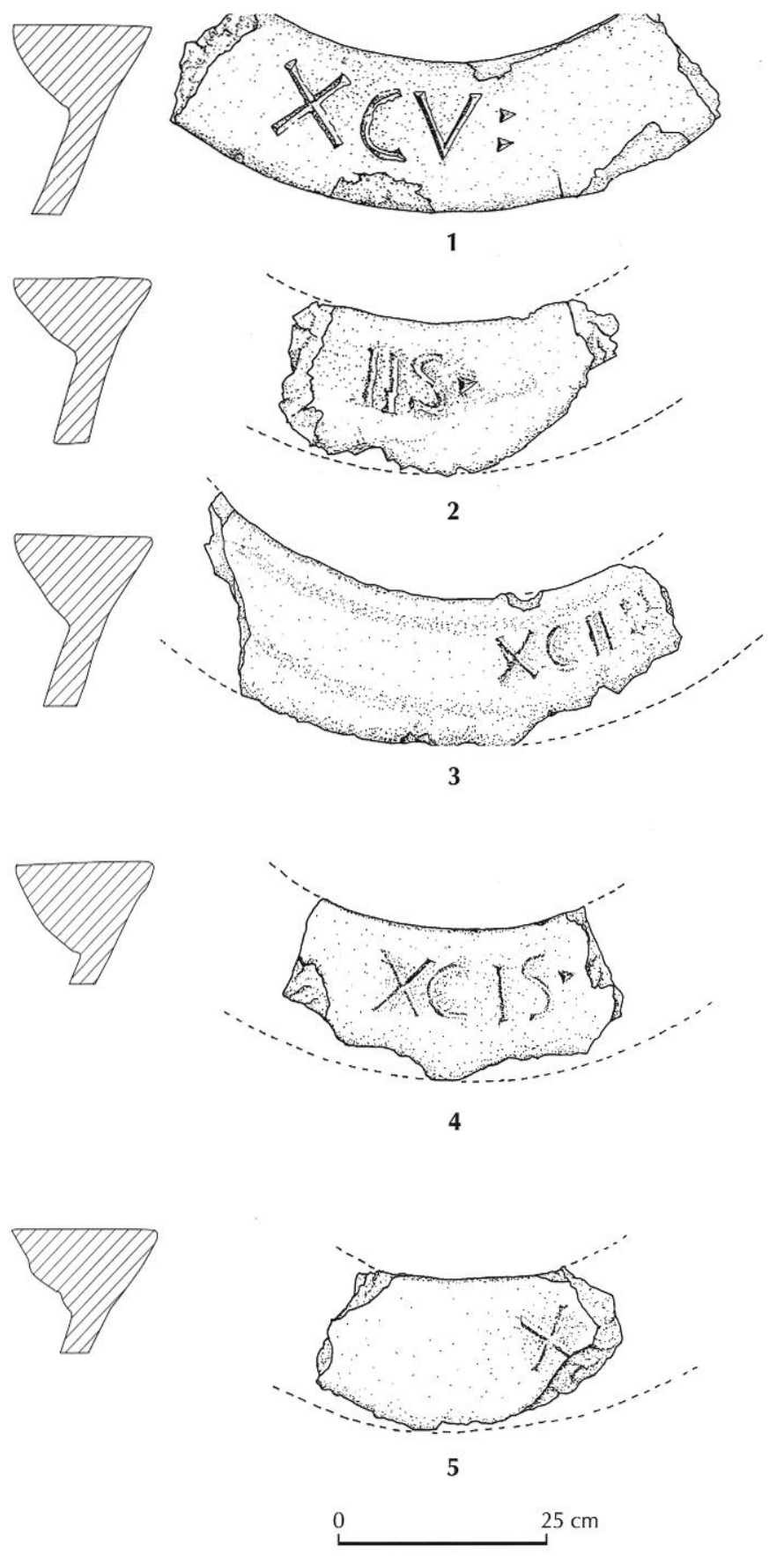

Fig. 57 - Inscriptions de capacité en urnae sur bords de dolia provenant du site du Molard à Donzère (dessin T. Odiot et C. Jung).

vaste vignoble attesté par les fouilles (Boissinot, Roger, à paraître).

Aux Malalones à Pierrelatte (fouille E. Henry), un établissement agricole se développe au cours du $\mathrm{I}^{\text {er }}$ et du $\mathrm{II}^{\mathrm{e}} \mathrm{s}$. Il est restructuré au III $^{\mathrm{e}} \mathrm{s}$. et abandonné à la fin du III $^{\mathrm{e}}$ ou au début du IV ${ }^{\mathrm{e}}$ s. (Henry, Linossier, 1997, p. 100). Le fouilleur a mis au jour des socles maçonnés qui pour- raient correspondre à des bases de pressoir (ibid., p. 102), mais il reste prudent sur cette identification, n'ayant pas trouvé d'autres indices qui attesteraient une production viticole (zone de stockage, fouloirs, etc.).

\section{LES DONNÉES DES PROSPECTIONS ARCHÉOLOGIQUES}

Lors des prospections pédestres systématiques réalisées dans la plaine de Pierrelatte et dans le sud du bassin de Montélimar, sept sites ont livré des fragments de dolia dont la quantité importante peut attester l'existence d'un lieu de stockage probablement lié à la production de vin (fig. 53).

Sur les sites de Sainte-Foy à Pierrelatte et de Ceras à Chantemerle-lès-Grignan, les dolia sont associés à des bassins. Ceux de Ceras, récemment dégagés, rappellent par leur taille ceux observés au Molard. L'ensemble de ces indices tend à renforcer l'identification de la fonction de transformation et de stockage viticoles de ces établissements.

\section{LES INSCRIPTIONS SUR BORDS DE DOLIA}

Des inscriptions de contenance imprimées avant cuisson sur les bords de dolia ont été retrouvées au Molard et à Saint-Gervais-sur-Roubion ; elles peuvent être interprétées comme la marque d'une extension importante de la viticulture en Tricastin (fig. 57). En effet, elles constituent, à notre connaissance, les seuls exemples trouvés en Narbonnaise. Le marquage de la capacité sur le dolium cru suggère une mesure de la capacité après fabrication tenant compte du retrait de l'argile à la cuisson.

Leur présence, à une courte distance de la vallée du Rhône et dans un secteur restreint, est exceptionnelle et pourrait être directement liée à un type d'exploitation tourné vers l'exportation ${ }^{150}$. Quoi qu'il en soit, le savoirfaire demandé pour ce type de production et, en conséquence, le coût de la mise en œuvre d'un cellier aussi grand que celui du Molard impliquent que le commanditaire ait disposé d'une grande fortune et qu'il ait eu en vue une production de masse.

150. Dans le cadre d'une bonne gestion du stock, il est nécessaire d'en connaître la capacité exacte. De plus, dans le cas de l'achat du vin " en cuves ", le négociant visitant la cuverie pourra apporter du crédit aux inscriptions imprimées avant cuisson. Des marques de contenance incisées sont également connues à Pontaix, Drôme (cf. supra, p. 87). 


\section{TERROIRS ET FAÇONS CULTURALES}

Malgré le nombre restreint des exploitations viticoles antiques connues en Tricastin, les données recueillies sur ces établissements apparaissent exceptionnelles. Par ailleurs, la découverte d'un grand nombre de fosses de plantation de vigne dans la plaine de Pierrelatte vient confirmer l'impression d'une viticulture intensive dans cette région (fig. 53). Les travaux archéologiques réalisés dans le cadre du TGV-Méditerranée ont permis de reconnaître, sur des dizaines d'hectares, des fosses de plantation de vigne, structures désormais bien connues dans toute la Narbonnaise (Boissinot, 1997; Monteil et al., 1999). De plus, les analyses paléoenvironnementales réalisées sur le remplissage des fossés de la centuriation $B$ d'Orange ont également mis en évidence l'extension de cette culture (Berger et al., 1999 ; Jung, 1999).

\section{LES FOSSES DE PLANTATION}

Les tranchées systématiques réalisées lors des travaux du TGV-Méditerranée ont mis au jour une série presque ininterrompue de fosses de plantation de forme carrée sur la commune de Lapalud (Vaucluse). Dans ces sondages, les fosses de plantation sont datées de l'époque romaine sans plus de précision (Alfonso, 1995), mais les fenêtres sur lesquelles des études approfondies ont été réalisées (site des Girardes et des Devès) permettent de les dater entre la fin du $\mathrm{I}^{\mathrm{er}} \mathrm{s}$. avant $\mathrm{J}$.-C et la fin du $\mathrm{I}^{\mathrm{er}} \mathrm{s}$. de notre ère (voir Boissinot, supra, p. 59-61; Gelot et al., 1996 ; Berger et al., 1999 ; Jung, 1999).

Sur la totalité du tracé, ces fosses présentent un module régulier. De forme carrée, elles mesurent entre $0,6 \mathrm{~m}$ et $0,7 \mathrm{~m}$ de côté et sont espacées de $1 \mathrm{~m}$ environ. Elles ont été retrouvées sur $2 \mathrm{~km}$ de tranchée de manière presque systématique dans chacun des sondages réalisés tous les $35 \mathrm{~m}$. Cette bande d'investigation de près de $100 \mathrm{~m}$ de large représente un vignoble attesté sur 200 ha environ, orienté selon la centuriation B d'Orange. Il est cependant impossible de savoir si l'ensemble de ces plantations est contemporain ou s'il correspond à des vignobles successifs. Les données recueillies lors des fouilles sur ces secteurs donnent des réponses différentes (fig. 58).

Le site des Devès montre deux plantations successives sur une superficie de $2000 \mathrm{~m}^{2}$ (Gelot et al., 1996). La première, dans la partie nord du site, est mise en culture au cours de la seconde moitié du $\mathrm{I}^{\mathrm{er}} \mathrm{s}$. avant $\mathrm{J}$.-C. ; elle est abandonnée au début du ${ }^{\text {er }} \mathrm{s}$. après J.-C. La deuxième parcelle est exploitée dans le courant du ${ }^{\text {er }}$ s. après J.-C. (Jung, 1999).

Les sondages du TGV-Méditerranée ont également montré la présence de probables plantations de vignes sur le site des Malalones, à Pierrelatte (Ferber, Ronco, 1996 ; Jung, 1999) (fig. 53). Ces fosses carrées, présentant un module de $1 \mathrm{~m}$ de côté, sont espacées de $1 \mathrm{~m}$ environ et sont, elles aussi, orientées selon la centuriation B d'Orange. Un fragment de dolium et de la céramique commune claire ont été retrouvés dans le remplissage de ces structures et du matériel du BasFmpire a été découvert dans un niveau de sol associé à ces fosses (Ferber, Ronco, 1996, p. 47). Même si ces fosses sont plus grandes que celles des sites des Girardes et des Devès, leur espacement paraît trop réduit pour qu'il puisse s'agir d'un verger. Par ailleurs, le niveau d'occupation antique auquel sont rattachées les fosses de plantation peut être mis en rapport avec l'établissement rural de Surel, retrouvé à moins de $200 \mathrm{~m}$ au nord.

\section{LES DONNÉES PALÉOBOTANIQUES METTANT EN ÉVIDENCE LA CULTURE DE LA VIGNE}

Une opération archéologique systématique sur les fossés de la centuriation $B$ d'Orange a également été menée lors des travaux du TGV-Méditerranée (Berger $e t$ $a l$. , 1999). Elle a permis de définir l'organisation du terroir tricastin et sa mise en culture durant l'Antiquité et le Moyen Âge, grâce à l'étude d'une centaine de fossés. L'analyse microstratigraphique réalisée sur le terrain a permis d'aborder un certain nombre de problèmes relatifs au fonctionnement des fossés et à l'évolution du découpage parcellaire (Berger et al., 1999 ; Jung, 1999). Par ailleurs, le cortège d'analyses paléoenvironnementales (anthracologie, palynologie, carpologie et malacologie) ${ }^{151}$, qui a été effectué dans chacune des unités

151. Analyse anthracologique : Laurence Rousseau sous la direction de Stéphanie Thiébault (Paris) ; analyses palynologiques : Solange FarbosTexier (CÉPAM, Valbonne) et analyses palynologiques et microfossiles non polliniques : Antonio Lopez Saez (Université de Madrid) ; étude carpologique : Laurent Bouby (AFAN, Toulouse); étude malacologique : Dominique Peyric avec la collaboration de Frédéric Magnin (Marseille) ; étude des phytolithes : Pascal Verdin (CÉPAM, Valbonne). 


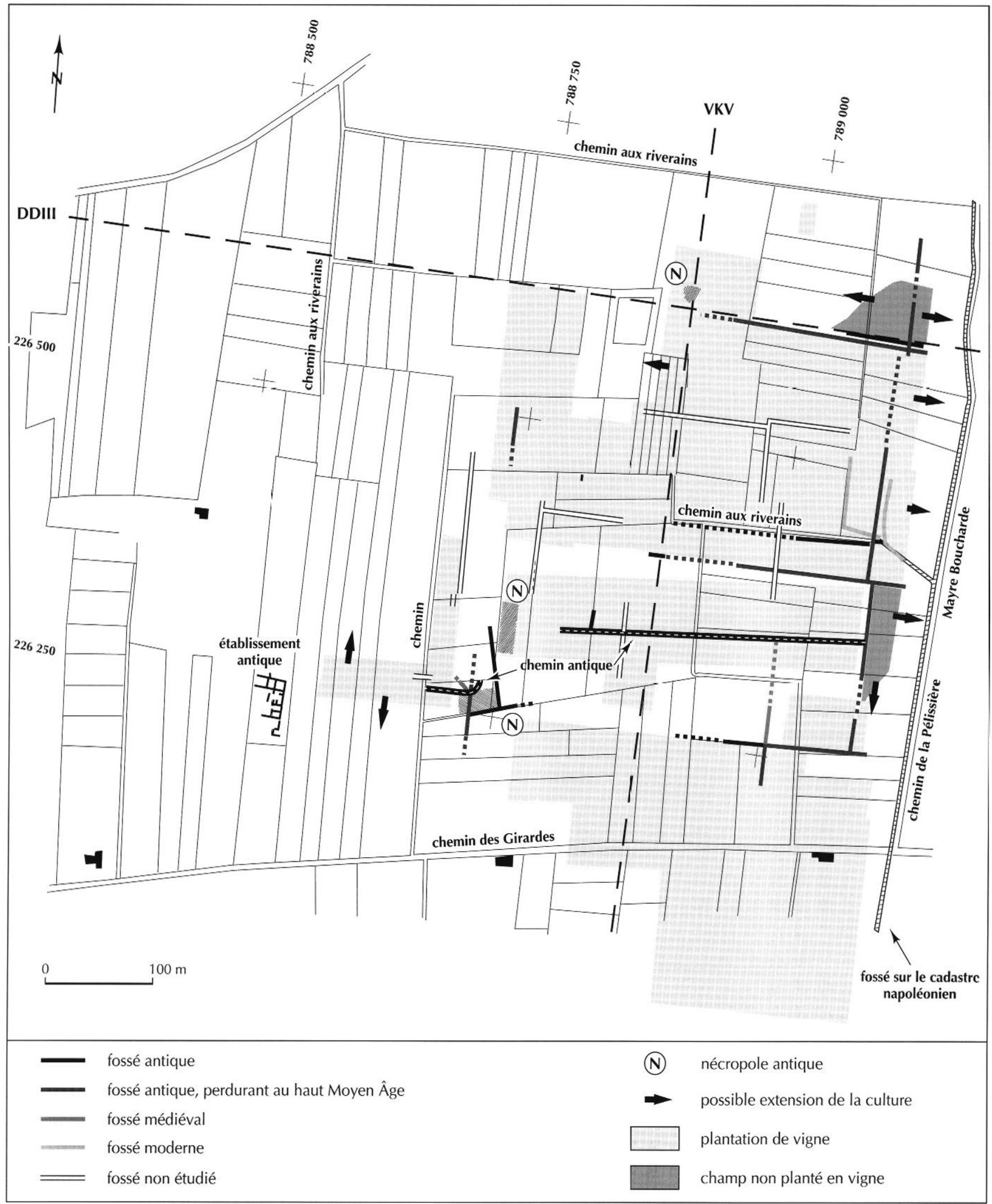

Fig. 58 - Plan du site des Girardes à Lapalud (Vaucluse). 
stratigraphiques constituant le remplissage de ces fossés, a permis de caractériser plus précisément le milieu dans lequel s'inscrivent ces structures fossoyées, de reconstituer les paléovégétations, de reconnaitre les types de cultures réalisées à proximité du fossé et également d'identifier des variations paléohydrologiques et climatiques. Bien qu'il soit délicat d'échafauder des hypothèses sur la restitution des paléopaysages par le biais d'analyses paléoenvironnementales trop peu nombreuses, ou à partir d'échantillons trop pauvres, la confrontation d'informations issues de différentes analyses peut confirmer ou infirmer les hypothèses émises sur les paléomilieux, c'est ce que l'on a tenté de faire au cours de cette opération.

\section{Plantation et domaine}

De nombreux fossés de la centuriation B d'Orange ont pu être étudiés sur les 30 ha du site des Girardes. L'étude de leur comblement a permis de comprendre le fonctionnement de ce terroir durant le premier millénaire de notre ère.

Les décapages et l'analyse stratigraphique ont permis d'observer que le réseau de fossés est postérieur au creusement des fosses de plantation du vignoble (fig. 58). Le fonctionnement synchrone du réseau fossoyé et des fosses de plantation est attesté par plusieurs éléments. L.es indices chronologiques montrent que le réseau de fossés est en place dès le début du ${ }^{\text {er }}$ s. après J.-C., voire à la fin du ${ }^{\mathrm{er}} \mathrm{s}$. avant J.-C. La présence de pollens de vigne dans les remplissages des fossés datés du $\mathrm{I}^{\mathrm{er}} \mathrm{s}$. de notre ère est également un bon indice pour attester la viticulture sur le site, puisque la vigne pollinise peu et ses pollens ne se dispersent pas sur de grandes distances (Lopez Saez et Farbos-Texier com. pers.) ${ }^{152}$. Enfin, la présence d'un pressoir au sein de l'établissement rural fouillé par Karine Roger (Boissinot, Roger, à paraître), à proximité immédiate du vignoble, peut constituer un lien entre cet

152. Il ne faut cependant pas négliger ici la possibilité de présence de Vitis vinifera (espèce de ripisylve) : des pépins de raisin et des pollens de vigne ont été retrouvés dans les sédiments colmatant ces fossés. La datation du premier remplissage d'un des fossés du site (Beta-109516: $2110 \pm 60 \mathrm{BP}$ ) donne une fourchette chronologique comprise entre 200 avant J.-C. et 70 après J.-C., lorsqu'on resserre la datation à 1 sigma, celle-ci demcurc encorc très large (165 avant J.-C./5 après J.-C.). La première phase de fonctionnement des fossés paraît donc se situer durant le $\mathrm{I}^{\text {er }} \mathrm{s}$. avant J.-C. La découverte de céramiques du $\mathrm{I}^{\mathrm{er}} \mathrm{s}$. avant établissement et le terroir avoisinant. La chronologie du site, qui est occupé aux I ${ }^{\mathrm{er}}$ et II ${ }^{\mathrm{e}} \mathrm{s}$., confirme l'exploitation de la vigne durant le Haut-Empire.

L'analyse du remplissage des fossés retrouvés sur le site atteste également la culture observée dans les basses plaines. Elle s'affirme surtout à partir de l'Antiquité tardive. L'exploitation pastorale de ces milieux apparaît alors dominante (Berger, 1995; Berger et al., 1999; Berger et al., 2001). La tendance se poursuit et même s'amplifie au cours du haut Moyen Âge, à la suite de l'abandon des réseaux drainants et d'une remontée des aquifères d'origine hydroclimatique, qui s'avèrent propices à l'extension de prairies humides et de zones palustres (Berger, Brochier, à paraitre). Une courte reprise des activités agricoles doit être cependant signalée à la fin de l'Antiquité, notamment dans la plaine d'Orange, sur les sites des Négades et des Crémades (Berger et al., 2001).

\section{LA SUPERFICIE DU VIGNOBLE ET LA TAILLE DES DOMAINES}

Les travaux archéologiques récents attestent l'existence de plantations de vigne dans le sud de la plaine de Pierrelatte sur de grandes superficies (fig. 53). Certains secteurs, où des décapages extensifs ont été réalisés, permettent d'estimer ponctuellement la taille des parcelles plantées, mais les données demeurent très lacunaires, sporadiques et parfois contradictoires comme l'illustrent celles recueillies sur les sites des Girardes et des Devès. $\mathrm{Si}$ la contemporanéité des sites viticoles semble établie, quand ils sont datés, au maximum de l'extension des traces de plantations, ces observations attestent la présence d'un grand vignoble dans la moyenne vallée du Rhône, au nord de la région où sont recensés les ateliers d'amphores liés aux domaines. Pour ce vignoble méconnu, il reste à aborder les problèmes de la super-

J.-C. dans le remplissage de deux des fossés confirme cette datation (Gelot et al., 1996; Berger et al., 1999). Le site des Devès permet de préciser l'évolution du paysage végétal de la plaine du Tricastin au cours des périodes historiques grâce aux analyses paléoenvironnementales systématiquement réalisées sur les sédiments remplissant les fossés du site. Ces résultats permettent de confirmer et d'affiner les évolutions et les tendances que l'on avait pu mettre en évidence sur le site des Girardes concernant l'extension de la vigne au cours du $I^{\mathrm{er}} \mathrm{s}$. de notre ère. 
ficie de ces centres de production sans oublier les problèmes de la nature du cépage, du type de plantation, de la qualité des sols et de la durée de stockage dans le cellier.

\section{LE RENDEMENT DU VIGNOBLE}

Une première question porte sur le rendement de la vigne en hectolitres/hectare, dans ce secteur géographique. Les agronomes antiques avancent des réponses variées. André Tchernia a fait le point sur la question et propose comme calcul de base une fourchette allant de 35 à $60 \mathrm{hl} / \mathrm{ha}$ (Tchernia, 1995b). Dans le cas du terroir du Molard les textes fiscaux dans la région de Pierrelatte, pendant l'ancien régime, attestent un rendement entre 8 et $12 \mathrm{hl} /$ ha (Peloux, 1985). Cependant, il s'agit de chiffres réli,lis dans le cadre de mesures de taxation qu'il faut donc majorer.

Nous choisirons donc, pour calculer la superficie possible de terres plantées en vignes dans le cadre du domaine du Molard, une fourchette allant de 15 à $30 \mathrm{hl} / \mathrm{ha}$. La capacité de stockage du cellier étant de l'ordre de $2500 \mathrm{hl}$, le vignoble aurait donc une superficie maximale de 167 ha et minimale de 71 ha. André Tchernia propose pour le même vignoble une fourchette allant de 41,6 à 85,4 ha, ce qui paraît tout aussi plausible.

\section{MODE DE PLANTATION, SOLS ET GESTION DU STOCK}

L'estimation proposée n'est valable que si le type de plantation de la vigne correspond à l'un de ceux décrits par Columelle (De l'agriculture, IV, 1) ${ }^{153}$, c'est-à-dire des ceps plantés avec un espacement de l'ordre de 3 pieds. Les résultats issus des fouilles archéologiques semblent le confirmer.

Il faut aussi évoquer le problème des types de sols cultivés. En effet, sur la plupart des sols alluviaux proches du Rhône, en zone parfois inondable, ou sur les sols hydromorphes de la vaste cuvette humide située à l'est de la plaine du Tricastin dans laquelle ont été repérées des fosses de plantation (site des Malalones), la vigne aura un rendement bien plus élevé que sur les coteaux ou les sols légers, calcaires et peu profonds des plateaux. Le

153. «[...] maxime probantur velut arbusculae brevi crure sine adminiculo per se stantes." rendement peut ainsi varier de 1 à 3 au minimum en fonction de ces éléments naturels.

L'ensemble de ces paramètres met en évidence la difficulté à proposer une superficie pour le vignoble d'un domaine. Si l'on peut émettre des hypothèses sur la superficie du terroir de la villa du Molard ou de celle du Fayn, grâce à la connaissance de leur chai, il n'en va pas de même pour les autres exploitations tricastines. André Pelletier a proposé une superficie moyenne de 100 jugères ( $25 \mathrm{ha}$ ) pour les exploitations du cadastre $B$ d'Orange (Pelletier, 1976). Compte tenu du caractère lacunaire des données fournies par les inscriptions, de la grandeur des chais connus, on peut envisager des domaines dont la taille pourrait atteindre voire dépasser 200 jugères : vignoble et cultures vivrières compris.

\section{COMMERCE : AXES ET CENTRES DE CONSOMMATION}

La place de la culture de la vigne dans cette région est illustrée par l'étendue des vignobles, les différents établissements viticoles reconnus et l'installation d'un chai d'une taille exceptionnelle. Ce développement pose le problème de l'insertion des domaines dans les réseaux de communication à petite, moyenne et grande échelle (fig. 59).

Les réseaux de communication en Tricastin durant l'Antiquité s'organisent à plusieurs niveaux. L'échelle locale est constituée par une série de chemins s'inscrivant dans le cadre du cadastre B d'Orange ou reliant directement les agglomérations secondaires entre elles. Ces itinéraires sont dédiés à la desserte des champs depuis les établissements ruraux et aux transports des personnes et des biens vers les agglomérations les plus proches.

Grâce à une étude régressive et morphologique du réseau routier relevé sur les cartes topographiques et les photographies aériennes, on a pu mettre en évidence un réseau régional, constitué par une série de voies probablement antiques qui aboutissent aux cités voisines (Jung, 1999). Leur datation relative repose sur le fait qu'elles évitent les centres de peuplement médiévaux et qu'elles sont associées à de nombreux sites antiques.

Enfin, au niveau provincial, deux axes majeurs permettent le commerce à grande distance : le Rhône et la voie d'Agrippa (Jung, Odiot, 1999). 


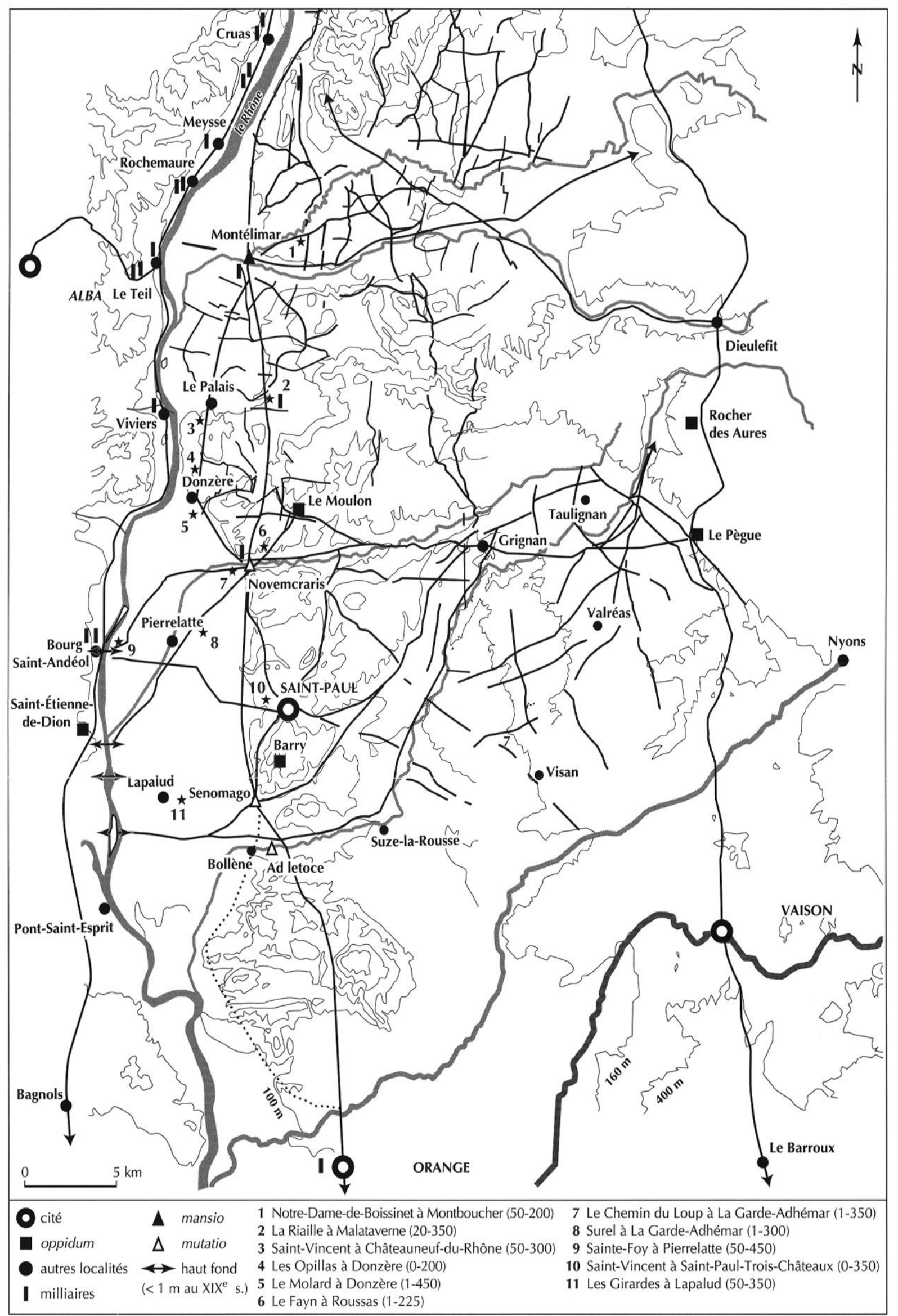

Fig. 59 - Carte des itinéraires antiques en Tricastin et Valdaine repérés par les analyses archéomorphologiques et localisation des établissements viticoles. 
Dans le cas du domaine du Molard, sa situation géographique peut illustrer son importance dans les relations économiques de la région. En effet, s'il est implanté à $3,5 \mathrm{~km}$ de la voie d'Agrippa, il se trouve en revanche le long d'un diverticulum de cette voie qui relie le Logis de Berre (Novem Craris, mutatio située sur la voie d'Agrippa) à l'habitat groupé de Donzère, avant de rejoindre la voie impériale au niveau de Montélimar. Sa proximité immédiate de l'agglomération portuaire de Donzère permet en outre d'envisager un commerce fluvial des productions de la villa. Cette hypothèse s'accorderait tout à fait avec l'absence d'ateliers d'amphores à proximité du site, qui pose un problème pour envisager une exportation de masse. En effet, le transport fluvial pouvait s'effectuer comme le transport maritime par " bateaux-citernes " chargés de dolia ${ }^{154}$ (Tchernia, 1986a) et les ateliers de mise en amphores pouvaient être fort éloignés. De plus, il faut envisager l'utilisation de tonneaux et d'outres dont les mentions régionales attestent l'existence. Ainsi l'inscription de Montélimar (CIL XII, 1, 1742), qui cite les utriculaires de Lyon et révèle des liens économiques entre les différentes agglomérations situées le long du Rhône, pourrait être versée à ce dossier.

Dans la même logique, le monolithe réutilisé comme linteau sur la façade méridionale de la chapelle romane de Saint-Pierre à Colonzelle (Drôme) est un élément méconnu (fig. 60). La scène, conventionnelle, représente une embarcation lourdement chargée de trois amphores, d'au moins quatre tonneaux contenant chacun environ 1300 litres (Marlière, à paraître) et d'un volumineux ballot. Il s'agit sans doute d'un fragment de monument funéraire (mausolée). Le type de représentation (scène commerciale) permet d'envisager que le défunt a pu être membre d'une corporation de nautes.

Les sites du Molard et de Sainte-Foy, à Pierrelatte, sont les plus proches du Rhône et des ports supposés. Les autres sites viticoles connus en Tricastin sont tous situés à proximité immédiate d'axes de communication locaux ou régionaux. Leur situation privilégiée au sein d'un réseau de communications régionales peut indiquer l'importance de la production viticole en Tricastin et sa commercialisation.

154. Il faut cependant préciser que ce moyen de transport n'est connu qu'en Italie et en Taraconnaise au cours du I ${ }^{\mathrm{er}} \mathrm{s}$. de notre ère.

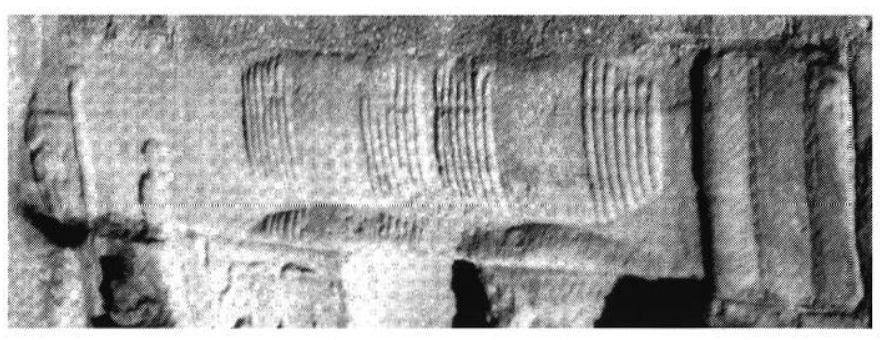

Fig. 60 - Scène de halage sur monolithe en réemploi dans la chapelle Saint-Pierre à Colonzelle (Drôme).

Aucun texte mentionnant le vin de Narbonnaise ne cite le Tricastin et les grands crus allobroges se situent à une centaine de kilomètres plus au nord. L'absence de textes mentionnant un cru ou un cépage dans le Tricastin est contrebalancée par la relative abondance des vestiges archéologiques. De même l'absence d'atcliers d'amphores dans la région qui reste à expliquer (lacunes des prospections, autre type d'emballage ?) est rééquilibrée par les inscriptions de Montélimar et de Rochemaure, Ardèche ${ }^{155}$ (en Helvie, à $15 \mathrm{~km} \mathrm{au}$ nord de Viviers) qui attestent qu'une partie de la société de la moyenne valléc du Rhône s'est spécialisée dans le commerce. Les relations entre Alba et Lyon et les échanges commerciaux dans la vallée du Rhône sont largement favorisés par l'appartenance au conseil municipal d'Alba de $M$. Inthatius Vitalis, négociant en vin et membre, entre autres, de la corporation des nautes de la Saône. La production viticole est somme toute banale dans le contexte de la moyenne vallée du Rhône. Au nord, les Allogroges possèdent un cépage qui fait leur renommée, et si les cépages tricastins ne sont pas connus, leur culture intensive contribue à l'essor économique de la Narbonnaise. L'existence de grands domaines viticoles dans cette région ne doit pas faire oublier les cultures vivric̀res assurant la subsistance des cités ; productions dont les traces ne sont pas facilement reconnaissables par l'archéologie. Toutefois la céréaliculture est clairement attestée au cours du HautEmpire dans le sud du Tricastin par les analyses carpologiques et palynologiques (Lopez. Saez, Bouby, in: Berger et al., 1999).

155. CIL XII, 2669. La dédicace funéraire retrouvée à Rochemaure sur la rive droite du Rhône montionne le collège des cupari Vocronnenses. 


\section{PROPOSITION D'ÉVOLUTION CHRONOLOGIQUE}

L'ensemble des résultats issus de l'archéologie et des analyses paléobotaniques permet de décrire l'évolution du paysage agricole du Tricastin dans l'Antiquité.

\section{LE HAUT-EMPIRE}

Le paysage durant le Haut-Empire semble globalement très ouvert; les analyses palynologiques réalisées sur les sites de plaine du Tricastin indiquent en effet un écho lointain des espèces forestières (moins de $10 \%$ ). En revanche, il semble que l'on puisse saisir des phases à caractère moins anthropisé qui peuvent correspondre à un abandon momentané de la culture sur certains sites ou dans un rayon plus large.

Durant le Haut-Empire, l'importance et la récurrence des phénomènes érosifs sur les versants et des accumulations sédimentaires alluviales dans les cônes détritiques ou les lits majeurs des cours d'eau du Tricastin et de la Valdaine confirment indirectement cette emprise agropastorale. La présence permanente de charbons de bois dans les séquences sédimentaires atteste un large défrichement des terroirs exploités. Dans l'ensemble de la région, l'identification de traits agricoles spécifiques d'une exploitation intensive des sols médio-rhodaniens montre une extension maximale des cultures et le faible degré de protection des sols en surface (Berger, 1995).

Durant cette période, la viticulture est bien attestée par les carporestes, les charbons de bois, les pollens conservés dans les fossés et par les vestiges archéologiques d'installations de production. Les analyses paléobotaniques montrent un développement de la viticulture dans l'ensemble du Tricastin : sites des Girardes et des Devès à Lapalud, du Duc à Mondragon et les hautes terrasses de la Valdaine. La présence de pollens de vignes signe la pratique de la viticulture locale.

Par ailleurs, des fosses de plantation de vigne ont été mises en évidence sur de vastes zones couvrant le sud de la plaine du Tricastin. La plupart de ces plantations remontent au début de notre ère et persistent durant le $\mathrm{I}^{\mathrm{er}} \mathrm{s}$. Les analyses palynologiques effectuées sur le site des Girardes semblent indiquer un arrêt de cette culture à la fin du I ${ }^{\text {er }}$ s. Aux Devès, une deuxième vague de plantations est réalisée dans le courant du
$I^{\text {er }}$ s., mais nous ignorons la durée de vie des vignes alors plantées.

Les établissements sur lesquels nous avons pu identifier des vestiges de pressoirs ou des lieux de stockage montrent également la place prise par cette culture dans l'ensemble de la plaine et des piémonts. Ainsi, on dénombre, grâce aux prospections et aux fouilles réalisées sur certains de ces établissements, une douzaine de sites ayant un lien avec la viticulture. Ceux-ci sont implantés au début ou au cours du $\mathrm{I}^{\mathrm{er}} \mathrm{s}$. de notre ère. Leur fonctionnement en liaison avec une activité viticole est attesté, le plus souvent, durant les deux premiers siècles après J.-C. Par ailleurs, la découverte d'un petit atelier d'amphores sur la commune de Saint-Just en Ardèche confirme l'importance de l'activité viticole dans ce secteur de la moyenne vallée du Rhône durant la période augustéenne (Laubenheimer et al., 1989).

La pratique d'une céréaliculture répandue dans l'ensemble de la plaine du Tricastin est attestée par les résultats des analyses paléobotaniques (Bouby, Lopez Saez et Rousseau, in: Berger et al., 1999), grâce aux pollens ou aux carporestes de céréales elles-mêmes ou aux carporestes de plantes adventices des cultures céréalières d'automne. Ces taxons ont été retrouvés sur les sites des Girardes et des Devès à Lapalud, des Brassières, des Ribauds et du Duc à Mondragon. Les analyses palynologiques réalisées dans le sud de la Valdaine sur les formations pédosédimentaires et les sites des piémonts marneux attestent également la culture des céréales dans le courant du ${ }^{\text {er }}$ s. après J.-C. et le milieu du II s. (Berger, 1995, p. 101).

$\mathrm{Si}$, sur certains sites, la présence simultanée de taxons de vignes et de céréales dans le remplissage des fossés a soulevé la question d'une culture en complants (vigne et céréales), l'écart très réduit entre les fosses $(1 \mathrm{~m}$ à $1,50 \mathrm{~m}$ ) et la présence de parcelles adjacentes vierges de fosses de plantation indiquant un autre type de culture éliminent cette hypothèse.

La culture du noyer peut également être envisagée au cours du Haut-Empire dans les piémonts du sud-est du bassin de Montélimar (Berger, 1995, p. 95). On ne peut l'attester avec certitude dans le Tricastin, où elle paraît plus discrète et peut correspondre à des arbres disséminés en lisière de champs, ou isolés en bordure de chemin. Enfin, signalons qu'aucun vestige de fosses de plantation d'arbre n'a été trouvé lors des fouilles réalisées en Tricastin. 


\section{L'ANTIQUITÉ TARDIVE}

Sur l'ensemble des sites étudiés, on remarque au cours de l'Antiquité tardive des moments de déprise culturale et de reconquête de la végétation. Sur le site des Brassières et des Ribauds à Mondragon, la récurrence des alluvionnements perturbe également la mise en culture de cette partie de la plaine.

Des taxons de mauvaises herbes ont été retrouvés fréquemment dans les niveaux de cette période. Ceux-ci attestent, de manière indirecte, la pratique de cultures céréalières, vivrières ou viticoles. Ils peuvent être divisés en deux groupes: les adventices d'automne, que l'on trouve dans les champs de céréales semées en cette saison, et les adventices de printemps et de cultures sarclées (légumineuses, jardins, vergers, vignes). On n'observe cependant pas de répartition chronologique ou spatiale distincte entre ces deux groupes, ce qui donnerait des indications sur l'évolution des types de cultures exploitées. Les deux groupes apparaissent souvent de façon concomitante dans une même station et dans un même niveau, la domination étant dans la plupart des cas en faveur des cultures sarclées de printemps (Bouby, in: Berger et al., 1999).

Les taxons d'olivier ne sont plus présents dans les diagrammes polliniques ou de manière très anecdotique. En revanche, la culture du noyer est encore attestée dans les piémonts du sud de la Valdaine (Berger, 1995, p. 95).

Les fossés du site des Devès à Lapalud présentent des taux élevés de pollens et de phytolithes de graminées dans les niveaux de l'Antiquité tardive. Les phytolithes de graminées à revêtement organique calciné pourraient indiquer des brûlis pastoraux. Le développement de prairies lié à un essor de l'élevage semble également attesté dans les cuvettes humides de la Valdaine à la fin de l'Antiquité. Les indices sont d'ordre pédologique et malacologique : développement de sols brun sombre de prairie associés à des spectres de malacofaune de prairie humide et de milieu palustre (Berger, 1995 ; Magnin, in : Berger, 1996).

Durant l'Antiquité tardive, les zones cultivées en vignes paraissent moins étendues que sous le HautEmpire; la plupart des sites à vocation viticole sont abandonnés à la fin du $\mathrm{II}^{\mathrm{e}} \mathrm{s}$. après J.-C. et les analyses paléobotaniques attestent également le recul de cette culture. Les fosses de plantation retrouvées associées à du mobilier du III ${ }^{\mathrm{e}}$ s. après J.-C. sur le site des Malalones à Pierrelatte sont les seuls vestiges archéologiques de champs de vignes de cette période. En Valdaine, J.-F. Berger évoque la présence de vignes durant l'Antiquité tardive (Berger, 1995, p. 95).

Si la viticulture est encore attestée par endroits, elle paraît nettement en recul par rapport à la phase précédente. Il semble donc qu'il faille envisager à cette période des terroirs plus diversifiés que ceux du Haut-Empire.

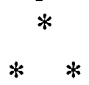

Le dossier sur la viticulture antique en Tricastin présente la particularité d'être documenté par un faisceau d'informations variées : archéologiques, paléobotaniques, iconographiques et épigraphiques. L'ensemble de ces données souligne la place éminente de cette culture durant le Haut-Empire. Elle semble s'étendre sur l'ensemble de cette région comme le montrent la répartition spatiale des établissements viticoles et les vestiges de plantation de vignes. Dès la période augustéenne et surtout dès le milieu du I I ${ }^{\text {er }} \mathrm{s}$. de notre ère, la moyenne vallée du Rhône semble être un espace d'investissements spéculatifs. De riches propriétaires plantent de vastes surfaces en vignes et font construire des villae spécialisées. Les hypothèses formulées plus haut sur les quantités produites montrent que cette production était probablement tournée vers l'exportation. Cependant le fait qu'aucun atelier d'amphores n'ait été jusqu'à présent retrouvé laisse penser que le vin était exporté en tonneau, sans soin particulier, et qu'il devait être de médiocre qualité. 